\title{
NOTAS SOBRE LA FORMACIÓN EN LA ESCUELA DE TRABAJO SOCIAL, UNIVERSIDAD DE COSTA RICA (UCR) DURANTE LAS DÉCADAS DE 1950 A 1980
}

\author{
Freddy Esquivel Corella \\ Profesor e investigador de la Escuela de Trabajo Social de la \\ Universidad de Costa Rica \\ San José, Costa Rica
}

Recibido 17-IX-2007 • Aceptado 13-XI-2007 • Corregido 17-XI-2007

En realidad toda fase histórica real deja huella de si en las fases posteriores,

que en cierto sentido llegan a ser el mejor documento. El proceso de desarrollo histórico es una unidad en el tiempo, por lo cual, el presente contiene todo el pasado, y en el presente se realiza del pasado todo lo que es "esencial» [...]

(Gramsci, 1999, p. 278)

\begin{abstract}
Resumen: El presente artículo, describe algunos de los cambios en los planes de estudios de la Escuela de Trabajo Social de la Universidad de Costa Rica (UCR), durante el periodo 1950-1980, décadas que forjaron una importante base de la trayectoria de madurez formativa en dicha profesión.

Los resultados del análisis, permiten identificar las principales presiones que influyeron en la formación de esas décadas, tanto por las coyunturas históricas nacionales, como por las contradicciones con la propuesta curricular de la Organización de Naciones Unidas (ONU), condiciones que llevaron posteriormente a la articulación con los debates propiamente del Trabajo Social latinoamericano.

A partir de ello, se destaca la importancia de reconocer esos esfuerzos como el mayor impulso crítico del siglo XX, en lo que a materia formativa en el Trabajo Social costarricense refiere.
\end{abstract}

Palabras claves: Trabajo Social, Costa Rica, formación, ONU, planes de estudio, reconceptualización.

\section{a) A guisa de introducción y reseña:}

El Trabajo Social, es considerado en la actualidad como una profesión de carácter internacional, la cual, mantiene diversas expresiones en el orbe, según los diferentes continentes, regiones o contextos de educación superior.

Su conceptuación, varía según las tradiciones académicas, éticas, políticas, filosóficas, teóricas y metodológicas.

Para efectos de este artículo, Trabajo Social es aprehendido como una categoría inserta en la división social y técnica del trabajo, que tiene en la política pública su principal base de institucionalización $\mathrm{y}$ legitimación. 


\begin{abstract}
The present article describes some of the changes in the curricula of the School of Social Work of the University of Costa Rica (UCR) during period 1950-1980, decades that forged an important base of the trajectory of maturity in this profession. The results of the analysis, allow to identify the main pressures that influenced in the formation of those decades, as much by the national historical conjunctures, as the contradictions with the curricular proposal of the Organization of United Nations (the UN), conditions that took later to the articulation with the debates properly of the Latin American Social Work. From it, the importance stands out of recognizing those efforts as the greater critical impulse of the XX century, related to the Costa Rican Social Work.
\end{abstract}

Key words: Social Work, Costa Rica, formation, UN, curricula, Reconceptualización.
A su vez, la materia prima de trabajo, se centra fundamentalmente en la "cuestión social", o sea en las resultantes de las tensiones históricas de las contradicciones dialécticas entre el capital y el trabajo; de tal manera, dicha profesión, encuentra en la órbita de las condiciones de reproducción social su principal eje de investigación e intervención.

En el particular costarricense, dicha categoría profesional, ha aportado tanto a su desarrollo institucional en el área de la protección social (Instituto Mixto de Ayuda Social, Caja Costarricense de Seguro Social, Patronato Nacional de la Infancia, Ministerio de Justicia y Gracia, Poder Judicial, Ministerio de Educación, universidades públicas, Instituto de Vivienda y Urbanismo, Instituto de Alcoholismo y Farmacodependencia, entre otras), así como en la planificación, operacionalización, evaluación, innovación, investigación, en expresiones vinculantes con la "cuestión social".

Entrando más de lleno en el desarrollo de la presente temática, es preciso recordar que la primera Escuela de Trabajo Social en Costa Rica, surgió fuera de la UCR, donde posteriormente ingresa a la Facultad de Ciencias Económicas; reseñando brevemente se cita:

En 1944 la Escuela se incorpora a la Universidad de Costa Rica, en la condición de escuela adscrita ante la necesidad de otorgarle carácter profesional y estatus a la profesión. Para 1947 pasa a departamento de la Facultad de Ciencias Económicas, hasta 1956, año en que se le otorga el rango de escuela anexa de la Facultad de Ciencias Económicas. Para el ingreso a carrera, se requería el Bachillerato en Humanidades o título de maestro normal. Se le otorgaba el título de Bachiller en Servicio Social a quien cursara dos años comunes con los estudiantes de ciencias económicas y tres años específicos de servicio social y la presentación de la tesis. A los alumnos especiales se les otorgaba un certificado de conclusión de estudios para ejercer como asistentes sociales (Molina y Sáenz, 1994, p. 101).

Para Guzmán (1973), ese ingreso careció de claridad sobre la formación y reflejó falta de compromiso con la realidad 
nacional, ya que estimuló más la búsqueda de un estatus académico, que un compromiso con las clases trabajadoras, agrega la autora:

El hecho de que se anexara a la Facultad de Ciencias Económicas y Sociales sin un previo cuestionamiento de lo que ello implicaba o implicaría en el futuro, incidió parcialmente en el estancamiento de la Escuela y por ende, de la profesión. Todo esto aunado a la orientación paternalista y asistencial de la misma y a las características personales y profesionales del profesorado y estudiantado, llevaron a la institucionalización de un Servicio Social descomprometido con su realidad, obstaculizando constantemente el verdadero cambió social que el país necesitaba (Guzmán, 1973, p. 53).

Se puede reconocer, tal y como cita Guzmán (1973), que desde los inicios de la fundación de la Escuela, ésta estaba incorporada en los esfuerzos de las fracciones hegemónicas, que requerían un fortalecimiento estatal ante las demandas de la "cuestión social".

La naturaleza de requerir cuadros profesionales, especialmente con un perfil operativo de la política social, se gestaba en un contexto histórico, donde el reformismo socialcristiano, trataba de dar respuestas a la crisis nacional, generada tanto por el desgaste del liberalismo heredado del siglo XIX, como por las serie de acontecimientos internacionales tales como la Primera y Segunda Guerra Mundial, la crisis de las Bolsas de Valores en 1929, y los escalonados enfrentamientos entre los Estados Unidos y la Unión Soviética.

En dicho escenario, aparece también la demanda de contar con una universidad estatal, desde luego, ese proyecto de casa de enseñanza superior, se edificaba en razón de ser otro insumo institucional que auxiliara los esfuerzos por desarrollar un proyecto de sociedad determinado, siempre en estrecho vínculo con el impulso al capitalismo local (sin omitir desde luego, las singularidades propias del contexto costarricense).

Por tanto, las carreras que ahí se localizaban respondían (con fuertes contradicciones) a la formación de cuadros profesionales que dieran soporte a dicho modo de producción; por ello, no parece extraño que el Trabajo Social, como resultado sincrético del capitalismo monopolista (monopolismo de Estado en Costa Rica), fuera "universitarizado" en una Facultad de Ciencias Económicas y Sociales, siendo dicha Escuela, la primera unidad académica de esas llamadas "ciencias sociales", que se ubican en los inicios de la UCR.

Lo anterior se destaca ya que la participación del Estado como interventor en las relaciones económicas y sociales era algo incipiente y poco estructurado en el país, a su vez, no se contaba con el recurso especializado, en el área de las ciencias sociales (más allá de la tradicional Facultad de Derecho), que apoyara las tareas de intervención en la "cuestión social".

Sin embargo, cabe afirmar, a diferencia de lo que argumenta Guzmán (1973), que el ingreso de la Escuela de Trabajo Social a la UCR, fue una decisión que luego impulsó el desarrollo profesional, mismo que la diferenció prontamente de la formación de otras experiencias centroamericanas, suramericanas, asiáticas y europeas.

Incluso, desde 1944 se hicieron esfuerzos por revisar las condiciones y orientaciones de la formación profesional, léase al respecto:

En 1944 se realizó, por parte de la Institución, un primer intento de discusión de los planes de estudio, dando participación a las entidades de bienestar social interesadas en la capacitación de su personal. Participaron en la discusión la Caja Costarricense de Seguro Social, el Patronato Nacional de la Infancia, la Secretaría de Trabajo y el Consejo Nacional de Prisiones (Mora, 1968, p. 20).

Por tanto, el surgimiento de la Escuela, su incorporación en la también recién fundada UCR, así como su incipiente estructuración con el resto de instituciones vinculadas con la políticas dirigidas a las "cuestión social", dieron las bases para que se constituyera una unidad académica con un desarrollo bastante particular, en el 
seno de una sociedad costarricense que se transformaba en materia política, económica, cultural, ideológica, haciendo a su vez algunas rupturas con el patrón de desarrollo del siglo XIX.
Algunos de los rasgos de la formación profesional, para esos años, se destacan en los siguientes cuadros:

Cuadro 1

Rasgos de la formación profesional en Trabajo Social, UCR.

(1942-1952)

\section{Rasgos de la Formación Profesional}

Teoría sin práctica académica

El plan de estudios no contempla la realización de algún tipo de práctica o el intento de articular las lecciones magistrales con la realidad social. Los estudiantes que ingresan son funcionarios de instituciones de bienestar social, las cuales demandan una capacitación técnica que les prepare y les permita una mayor clarificación [...] La organización y orientación de los cursos, que establece el plan de estudios, se instituye de acuerdo a la utilidad práctica que demanda el trabajo institucional [...] La organización académica en este momento muestra un desempeño satisfactorio a nivel institucional y hay una estrecha coordinación entre demanda y oferta profesional. El contacto con la realidad se hace a expensas de la práctica institucional que realizaban como funcionarios los estudiantes.

Elaboración propia a partir de Campos, 1992.

Cuadro 2

Estructura Académica de la Escuela de Trabajo Social

(1942-1953)

\begin{tabular}{l|l|l}
\hline \multicolumn{1}{c|}{ Objeto de la profesión } & \multicolumn{1}{c}{ Propósito de la Escuela } & Organización Curricular \\
\hline $\begin{array}{l}\text { Contribuir al ajuste del indi- } \\
\text { viduo al medio, armonizar las } \\
\text { condiciones de vida del hombre } \\
\text { y sociedad. }\end{array}$ & $\begin{array}{l}\text { Proporcionar a las instituciones de protec- } \\
\text { ción social, personal idóneo, para el ejerci- } \\
\text { cio de la acción social. }\end{array}$ & $\begin{array}{l}\text { Coordinación efectiva entre } \\
\text { la demanda y oferta profe- } \\
\text { sional. }\end{array}$ \\
\hline
\end{tabular}

Fuente: Molina y Romero, 1992.

De esta manera, se puede resumir que décadas antes del Estado empresario (1950-1980) en Costa Rica, ya se había iniciado y desarrollado el análisis de la formación en Trabajo Social, cada vez más vinculante con la institucionalidad universitaria nacional, la cuál, también se encontraba dando los primeros pasos de su desarrollo (Esquivel, 2007).
Como se aprecia, la crítica y la autocrítica de la formación profesional ha sido característica del proyecto formativo original de la Escuela, sin embargo, su naturaleza y objetivos no necesariamente se vinculaban más allá de una orientación reformista.

Sin desestimar lo antes señalado, se considera que la experiencia heredada 
proyecta una unidad académica con preocupaciones que se movieron en la necesidad de:

- Independizarse de las propuestas educativas en Trabajo Social derivadas de la ONU.

- Intentar dar respuesta al contexto nacional y latinoamericano.

- Plantear la interlocución universitaria con distintas áreas del conocimiento.

- Participar en organismos regionales que buscaban renovar la educación superior de Trabajo Social en Latinoamérica.

El principal eje crítico que caracterizó este debate fue el tema de la relación teoría práctica, tensión de relevancia histórica para una profesión de naturaleza interventiva.

Aunado a ello, la crítica a una fuerte dependencia laboral del Estado capitalista, fue blanco de reflexión, considerando en ese momento que el significado real de la profesión adquiría sentido, primordialmente, al vincularse con las llamadas organizaciones populares, o no dependientes del Estado, quienes supuestamente, no representaban intereses antagónicos.
Sin duda, este período se caracteriza por ser uno de los momentos más intensos de reflexión educativo-político que se identifican en la historia del Trabajo Social costarricense, cuyo impacto ha dejado su impronta hasta inicios del siglo XXI.

\section{b) Cambios en los planes de estudio}

El plan de estudios de 1954, fue el segundo esfuerzo de actualización formativa en el Trabajo Social costarricense, el mismo, incluyó cursos de Economía, Sociología (rural y urbana), Administración Pública, Historia económica y social, Introducción al Servicio Social, Métodos de Servicio Social, Bienestar familiar, Psicología general y aplicada, Psiquiatría, Higiene mental, Medicina social, Criminología, Derecho positivo, Legislación, Sindicalismo, Estadística general, Organización de la comunidad, y tres cursos de práctica.

Aunado a ello se caracterizó por lo siguiente:

Cuadro 3

Estructura Académica de la Escuela de Trabajo Social

(1954-1964)

\begin{tabular}{|c|c|c|}
\hline Objeto de la profesión & Propósito de la Escuela & Organización Curricular \\
\hline $\begin{array}{l}\text { Contribuir al ajuste del individuo } \\
\text { al medio, armonizar las condiciones } \\
\text { de vida del hombre y sociedad } \\
\text { (Se mantiene como en 1942) }\end{array}$ & $\begin{array}{l}\text { Proporcionar a las instituciones } \\
\text { de protección social, personal } \\
\text { idóneo, para el ejercicio de la } \\
\text { acción social } \\
\text { (Se mantiene como en 1942) }\end{array}$ & $\begin{array}{l}\text { Se introducen cursos prácticos. La } \\
\text { teoría y la práctica está a cargo de } \\
\text { profesores diferentes } \\
\text { (Innovación de la época) }\end{array}$ \\
\hline
\end{tabular}

Fuente: Molina y Romero, 1992.

Cuadro 4

Rasgos de la formación profesional en Trabajo Social, UCR.

(1952-1965)

\begin{tabular}{l}
\hline Rasgos de la Formación Profesional \\
\hline Teoría y práctica, una unidad \\
{$[\ldots]$ se incorporan los cursos prácticos. La práctica académica se fundamenta en la investigación, tratamien- } \\
to a individuos y familias. Se conocen profundamente situaciones individuales, se interviene mediante la \\
asistencia a sus necesidades inmediatas y a la búsqueda de las potencialidades que poseen los usuarios para \\
enfrentar y resolver sus problemas. Las acciones emanan de la incorporación de los estudiantes a programas \\
institucionales, los cuales se constituyen en el medio, entre los estudiantes y los usuarios de los programas \\
institucionales.
\end{tabular}

Elaboración propia a partir de Campos, 1992. 
Sumado a ello, en los años en que se inicia Trabajo Social en la Universidad de Costa Rica con el rango de Escuela (1957), se establece una reestructuración bajo un nuevo reglamento que contiene entre otras cosas:

- Incorporación de la Cátedra de Organización Económica y Social de Costa Rica.

- Búsqueda continua de una articulación teoría-práctica.

- Una educación de tres años; y que exigía una defensa de tesis para otorgar el título de Trabajador/a Social, donde con una ampliación del plan básico y con determinados requisitos, se podría optar por una licenciatura. (Véase la diferencia entre título profesional y académico que se expresó en ese tiempo).

- 900 horas de práctica y 4 horas mensuales de supervisión a cada alumna (o).

- Ingreso a la carrera, regulado por una entrevista previa de ingreso.

- Autorización de los primeros textos propios de la Escuela de Trabajo Social ${ }^{1}$.

- Incorporación de un año de Ciencias y Letras.

- Un nuevo reglamento de evaluación.

- Necesidad de promover la carrera de Trabajo Social en la población costarricense.

- Brindar el título sin rango académico, a aquellos (as) estudiantes que hayan cursado el plan, sin el año de Ciencias y Letras.

Antes del siguiente cambio de mayor envergadura ${ }^{2}$, mismo que se llevó a acabo en 1965, cabe resaltar que para los años de 1960 a 1964 se realizaron varias modificaciones en los programas de estudio y en el reglamento de evaluación; a fines de 1963 , la Escuela se logra desligar totalmente de los programas de la Facultad de Ciencias Económicas, por lo que sus alumnos/as llenaban créditos con materias que fueran de su libre escogencia, a excepción del curso de ética, que era impartido siempre por esa Facultad. (Tema interesante de recuperar en el marco del debate ético-político contemporáneo en Trabajo Social).

Una consultora de la ONU, que había evaluado la formación de esos años destaca lo siguiente en su "Informe sobre la Escuela de Servicio Social de la Universidad de Costa Rica (1964)":

[...] una enseñanza que no esté respaldada por la investigación, viene pronto a ser caduca y se fosiliza rápidamente. Por lo demás, el Servicio Social moderno exige una renovación continua que supone actividades de investigación. Muchas veces, los institutos no pueden distraer el trabajo cotidiano, ni el personal, ni los recursos indispensables a la investigación. Por este motivo esta función debe ser desempeñada por la Escuela [...] Una de las labores de la Universidad es reflexionar con serenidad y objetividad sobre los problemas de la Nación. La Escuela de Servicio Social debe reflexionar sobre los problemas sociales de Costa Rica. No puede por supuesto, por sí sola, definir la política social de un país, pero faltaría a su vocación si no participara en esta definición, trayendo al gobierno y a los diversos institutos un conocimiento de los problemas nacionales, de las realizaciones y experiencias comprendidas en otras partes del mundo, una capacidad para ver más allá de las necesidades inmediatas [...] pasó el tiempo en que el Trabajador Social podía contentarse con una cultura rudimentaria y con una formación superficial, que podía servirle un poco para todo, transformándolo, en cierto modo, en "mozo" de la caridad privada o de los servicios públicos de asistencia [...] El Trabajador Social es hoy día un especialista, practicante de las Ciencias Sociales. Solamente puede uno llegar a ser un buen Trabajador Social si ha adquirido una sólida formación básica en el campo de las ciencias sociales [...] Pensamos que dentro de algunos años, la Escuela debería reclutar a sus alumnos entre los titulados dentro del Centro de Ciencias del Hombre [Departamento de Ciencias del Hombre] (Labebens, 1964, pp. 12, 13, 15, Citado en Mora, 1968, pp. 31 y 32$)^{3}$

Al parecer, las recomendaciones de Labbens eran realmente interesantes para la época, pero las condiciones locales aún en la academia, y con el sincretismo que matizaba a la profesión, no permitía un avance en esa discusión.

Posterior a la evaluación de dicho organismo internacional, la Escuela aumentó requisitos de ingreso, por ejemplo: 
La selección de los estudiantes se efectúa a base de la aprobación de las materias generales de Ciencias y Letras, a saber, castellano, Filosofía e Historia de la Cultura. Además, deben realizar una serie de pruebas sicométricas en el Departamento de Bienestar y Orientación de la Universidad, que se refieren a aspectos de capacidad general, personalidad, interés por la profesión y grado de socialización. El último requisito es una entrevista con un profesor de la Escuela nombrado al efecto y en la cual se trata de determinar la situación personal y familiar del futuro alumno en cuanto pueda influir sobre el curso normal del proceso de formación profesional (Mora, 1968, pp. 34 y 35).

También, cambiaron contenidos de cursos, y se incluyeron nuevas materias, hubo preocupación por la investigación, y la proyección externa de la Escuela hacia la sociedad costarricense, se solicitaron nuevos docentes, se estimuló un programa de becas en las instituciones, para que los/as nuevas alumnos/as pudieran seguir estudiando, así como independencia por parte del decanato de Ciencias Económicas, quedando a cargo la Escuela del Concejo de Profesores y la Dirección de ésta propiamente.

La venida de la ONU y sus recomendaciones no estuvo libre de críticas, sobre este particular léase lo siguiente:

Si retornamos al análisis anterior sobre el Servicio Social tradicional, nos damos cuenta de que dichos programas de asesoría pretendían institucionalizar un Servicio Social que protegiera los intereses de los países del centro. La problemática que se establece en la Escuela, cada vez con mayor fuerza, es la de dominación y dependencia. Se carece de un cuestionamiento y análisis crítico objetivo en relación a estas reco- mendaciones y de los móviles detrás de las mismas. La Escuela se vanagloria de los esfuerzos realizados por llevar a la práctica y en el menor tiempo posible, estas proposiciones, pero su cuerpo docente y el alumnado no ven la posibilidad de efectuar ellos mismos las reformas sin necesidad de la asesoría extranjera (Guzmán, 1973, pp. 54 y 55).

Para 1965 se da un cambio en la estructura curricular, donde la llamada sistematización de la "metodología de la práctica" toma relevancia, igualmente se incluyen cursos en que los/as sujetos/as con que se trabajan son personas, grupos y comunidades; también se establecen intervenciones formativas en instituciones, manteniéndose siempre el desvelo de la búsqueda de la integración de la relación teoría-práctica.

El plan de 1965 incluía cursos de Biología, Matemáticas, Castellano, Filosofía, Historia de la cultura, Historia de las instituciones, Psicología (normal y anormal, del desarrollo de la personalidad, social), Sociología (familiar, para el cambio social, rural y urbana), Economía, Servicio Social (introducción, grupal), Derecho (familiar, social), organización económica y social de Costa Rica, Administración (programas de bienestar social), Psiquiatría (teoría y práctica), Investigación social, Organización comunal, Ética, y cursos optativos como Planificación económica y social, Medicina Social, Criminología y cuatro cursos de práctica, entre otros.

Sus rasgos básicos se resumen de la siguiente forma:

Cuadro 5

Estructura Académica de la Escuela de Trabajo Social

(1965-1972)

\begin{tabular}{|c|c|c|}
\hline Objeto de la profesión & Propósito de la Escuela & Organización Curricular \\
\hline $\begin{array}{l}\text { Contribuir al ajuste del } \\
\text { individuo al medio, armoni- } \\
\text { zar las condiciones de vida } \\
\text { del hombre y sociedad } \\
\text { (Se mantiene como en } \\
\text { 1942) }\end{array}$ & $\begin{array}{l}\text { Capacitar al estudiante para ejercer la pro- } \\
\text { fesión del Servicio Social, fortalecer la con- } \\
\text { ciencia de responsabilidad profesional y su } \\
\text { obligación de contribuir al bienestar de la } \\
\text { humanidad } \\
\text { (Innovación de la época) }\end{array}$ & $\begin{array}{l}\text { Se incorporan los cursos } \\
\text { de método de caso, grupo y } \\
\text { comunidad. La práctica era } \\
\text { institucional en todas sus } \\
\text { expresiones. } \\
\text { (Variación de la época) }\end{array}$ \\
\hline
\end{tabular}

Elaboración propia a partir de Molina y Romero, 1992. 
Cuadro 6

Estructura Académica de la Escuela de Trabajo Social

$(1965-1975)$

Rasgos de la Formación Profesional

Teoría y Práctica, dos dimensiones paralelas

En 1965, se consolida la metodología de intervención individual con grupos (tanto incipientes, como consolidados) con proyección a la comunidad. Se incorpora el proceso de investigación social a la práctica. En 1968, las prácticas privilegian el trabajo comunal, y la participación de los grupos en acciones en beneficio de las comunidades. El cuestionamiento de la formación académica mediante la práctica se expresa en dos momentos diferenciales: Uno de aproximación sensible a las comunidades seleccionadas como centros de práctica [...] y otro de estudio de literatura sobre nuevos modelos de intervención en la realidad aportados por el llamado "Movimiento de Reconceptualización" que irradiaba influencia de los países más industrializados de América del Sur, como Argentina, Brasil, Chile.

Elaboración propia a partir de Campos, 1992.

Haciendo una revisión crítica de la formación de la época se señala lo siguiente:

La orientación del Servicio Social del período- [...], responde a la percepción particular que de las necesidades sociales tienen las Instituciones de Bienestar Social, a saber, necesidades que tienen su origen en situaciones particulares al individuo y no en situaciones estructurales, por tanto, las formas de abordarlas va a ser a ese nivel individual y en fases posteriores a nivel de grupos, por tanto, la formación profesional va en esta misma línea atendiendo realmente a la demanda institucional de personal capacitado. Esta afirmación se cimienta en: - La aplicación dominante del método de Caso en las instituciones, y, el énfasis dado en la Escuela a cursos teóricos de Psicología, Psiquiatría, Legislación Social y al Método de Caso Social Individual, con una clara concepción atomista de la realidad, adoptada por las Instituciones que reclutan a los profesionales en Trabajo Social, se caracterizan ambas por una acción asistencial (Campos et al., 1977, p. 171).

En el año de 1968, que es cuando aún se mantiene el currículo antes señalado, se afirmaba que el programa de estudios era muy completo y estaba estructurado de acuerdo con recomendaciones de las Naciones Unidas, lo cuál, se afirmaba, establecía un sólido prestigio en América Latina para dicha Escuela (Mora, 1968).

Sin embargo, para otros sectores, ese tipo de dependencia internacional, era principalmente una forma de apoyar un
Estado que buscaba mayor control social; por lo que se alegaba que la situación de Costa Rica (y en otras partes donde la ONU se había colocado en la asesoría formativa en Trabajo Social) se mantenía "desconectada", de las verdaderas condiciones de explotación que asolaba a la región latinoamericana.

Sobre esos años se puede destacar también lo siguiente:

La preparación académica de la Escuela no permitía al estudiante adquirir los instrumentos necesarios para enfrentar su realidad, pese a que desde 1968 el programa de estudios incluía materias fundamentales y complementarias [...] Pero ni la formación académica fue la adecuada ni se le permitió al estudiante adquirir los instrumentos para que analizara su realidad objetivamente (Guzmán, 1973, p. 57).

Sumado a ello, se destaca que las condiciones históricas en los años sesentas eran realmente complejas, léase al respecto:

A finales de la década del 60, principios de la siguiente década se inicia un proceso que bien podemos calificar como una ruptura en la forma de percibir los objetivos y el objeto del Trabajo Social. En esta ruptura juega un papel importante el movimiento de reconceptualización del Servicio Social que se planteaba, en el cono sur de América Latina. Es importante señalar que el ascenso de los militares al poder en América del Sur, desató una feroz y salvaje represión, obligando al exilio involuntario de muchos 
profesionales entre ellos trabajadores sociales. Los cuales felizmente para nuestro país vienen y dan sus aportes al trabajo social. Otro elemento a tomar en cuenta es la participación de costarricenses en el tercer seminario de trabajadores sociales, celebrado en Quito en 1971 (Araya et al, 1986, pp. 40 y 41).

Por otro lado, cabe también destacar que la Escuela de Trabajo Social (UCR) ha tenido históricamente Jornadas de Evaluación sobre la formación profesional, las cuales, han conducido a cambios en sus planes de estudios, surgiendo modificaciones curriculares, sin omitir desde luego, las limitaciones históricas para romper con las bases sincréticas que la han sustentado desde su origen en el país.
Dicho sincretismo se constituye por una serie de influencias filosóficas, éticas, políticas, ideológicas y culturales, que se traslapan históricamente, sin necesariamente ser definidas por sus cuadros académicos; se trata por tanto de una herencia de entramados (algunos bastantes contradictorios), que no fueron puestos en cuestionamiento, sino, parcialmente, y hasta momentos más particulares como la Reconceptualización que caracterizó a dicha profesión en los años setentas.

El siguiente cuadro ilustra sobre los alcances de dichas Jornadas:

Cuadro 7

Jornadas de reflexión y evaluación de la Escuela de Trabajo Social, UCR

(1971-1975)

\begin{tabular}{|c|c|c|c|}
\hline Fecha & Jornada & Tema & Resultado \\
\hline Octubre, 1971 & I & $\begin{array}{l}\text { El/la Trabajador/a Social que el } \\
\text { país necesita. }\end{array}$ & $\begin{array}{l}\text { Decisión de realizar cambios } \\
\text { curriculares. }\end{array}$ \\
\hline Agosto, 1972 & II & $\begin{array}{l}\text { Papel del Trabajador/a Social. } \\
\text { Papel de la Escuela en la } \\
\text { Universidad de Costa Rica y en } \\
\text { la sociedad. } \\
\text { Definición de la enseñanza y } \\
\text { método pedagógico. }\end{array}$ & $\begin{array}{l}\text { Propuesta de Plan de estudios } \\
\text { que significó una ruptura para- } \\
\text { digmática. }\end{array}$ \\
\hline Octubre, 1973 & III & $\begin{array}{l}\text { La integración teórico-práctica } \\
\text { en el currículo. } \\
\text { Métodos participativos en la } \\
\text { enseñanza. }\end{array}$ & $\begin{array}{l}\text { Recomendaciones en torno a la } \\
\text { integración en la docencia, la } \\
\text { investigación y la acción social. }\end{array}$ \\
\hline Julio, 1974 & $\begin{array}{l}\text { IV Seminario } \\
\text { Latinoamericano } \\
\text { de Escuelas de } \\
\text { Servicio Social, } \\
\text { con Sede en Costa } \\
\text { Rica }\end{array}$ & $\begin{array}{l}\text { Relación teoría-práctica y } \\
\text { medios para lograrlo. } \\
\text { Estructura organizativa de las } \\
\text { Escuelas. } \\
\text { Relación Escuelas-Instituciones } \\
\text { de Bienestar Social. } \\
\text { Sistematización de la práctica. }\end{array}$ & $\begin{array}{l}\text { Recomendaciones en todas las } \\
\text { áreas. Se destaca lo referente } \\
\text { al Taller como método pedagó- } \\
\text { gico y propiciador de la triple } \\
\text { integración: Teoría-Práctica, } \\
\text { Investigación, Docencia-Acción } \\
\text { Social, Estudiantes-Docentes y } \\
\text { grupos populares. }\end{array}$ \\
\hline $\begin{array}{l}\text { Noviembre, } 1974 \\
\text { Junio, } 1975\end{array}$ & IV & $\begin{array}{l}\text { Currículo cuya base fuera la } \\
\text { integración y la perspectiva } \\
\text { crítica. }\end{array}$ & $\begin{array}{l}\text { Recomendaciones para rees- } \\
\text { tructurar el plan de estudios } \\
\text { 1976, sobre la base de la inte- } \\
\text { gración y el Taller como método } \\
\text { pedagógico. Determinación de } \\
\text { las competencias de la estruc- } \\
\text { tura organizativa de la Escuela. } \\
\text { Recomendaciones en torno a } \\
\text { la interdisciplinariedad en la } \\
\text { formación. }\end{array}$ \\
\hline
\end{tabular}

Fuente: Comisión de Autoevaluación y Autorregulación de la carrera de Trabajo Social, 1998. 
Retomando a Guzmán (1973), se señala que en 1971 y luego en 1972 (I y II Jornadas de Trabajo Social) profesores y estudiantes se abocaron a la realización de una evaluación crítica de los programas de estudio, dándose planteamientos de una reestructuración total en todos los niveles, ello, sin solicitar orientación internacional; dichas actividades, continuaron buscando la integración y relación de la teoría-práctica, se agrega al respecto:

Pero las deficiencias en la práctica profesional y la desintegración del gremio continúan fuera de la Escuela. La resistencia al cambio de parte de gran número de profesionales, ha ocasionado en varias instancias la formación de dos bandos contrarios. Y está por demás decir la misma situación se presentó y se presenta en la Escuela (Guzmán, 1973, p. 59).

La autora, deja plasmada en esa cita, las tensiones que se localizaban en la categoría profesional, ya que sectores de diferente orientación, apoyaban o rechazaban una tendencia crítica en el Trabajo Social.

Ahora bien, como esfuerzo síntesis de lo expuesto hasta ahora en este apartado de cambios en los planes de estudio, se destaca que:

- Las reflexiones sobre los cambios curriculares, fueron característica permanente de la formación académica universitaria del Trabajo Social costarricense en las décadas en estudio.

- $\quad$ Es evidente que el ingreso al seno universitario, posibilitó mayores insumos formativos para los profesionales y las profesionales (lo que no asegura necesariamente una vertiente crítica), igualmente, el tema de las prácticas pre-profesionales toma un lugar particular que no se había identificado antes.

- El acceso a la certificación profesional y académica, la selección previa para cursar la carrera (innovación en la evaluación formativa), elaboración y publicación de los propios textos nacionales, así como el establecimiento paulatino de los llamados estudios en ciencias y letras, propios de la estructura de la UCR de esos años, fueron una conquista que no fue un simple resultado del azar.

- La búsqueda de autonomía de asumir responsabilidad de independencia para tomar decisiones sobre la formación, fue otra de las características que se colocan en esta época, ello, a pesar de la presión que se recibía de algunas instituciones que antes habían patrocinado la fundación de la Escuela.

- Desde esas décadas, la Escuela de Trabajo Social se propuso ser una instancia que decidiera sobre sus propios asuntos formativos, lo cual implicó una madurez organizativa que no solamente se fue mejorando, sino que incluso rebasó los marcos universitarios (especialmente al incorporar el eje de la interdisciplinariedad en las experiencias de los llamados "Talleres", los cuáles consistían en prácticas integradas por diversos niveles de carrera, especialmente en zonas rurales, bajo la supervisión de equipos de docentes de distintas disciplinas).

- La formación, por tanto, fue dando evidencias de que no había satisfacción ni deseo de continuar bajo el rumbo de la ONU, dando más bien un giro por la preocupación propiamente latinoamericana, con aires de crítica a la propia historia formativa de origen norteamericano (algunas veces de tono radical y poco fundamentado).

\section{c) Reconceptualización en Trabajo Social y la autocrítica formativa:}

Como es conocido en la literatura especializada en Trabajo Social, en los años 19651975 existieron las condiciones históricas en 
América Latina, para el surgimiento del llamado Movimiento de Reconceptualización.

Aproximadamente, fue en 1965 bajo el I Seminario Regional Latinoamericano de Servicio Social, en Porto Alegre, Brasil, cuando se puede afirmar que inició formalmente dicho Movimiento, el cual, tenía como intención superar la visión tradicional (estadounidense) de la profesión, y construir un nuevo proyecto de Servicio Social en América Latina.

En esos años, el continente presentaba una serie de condiciones históricas, matizadas por las políticas imperialistas de los gobiernos estadounidenses, quienes alentaron el establecimiento de dictaduras especialmente en los países del cono sur.

Según Retana (2002) y Araya et al. (1986), algunas de las características de la Reconceptualización, que se dieron en suelo nacional fueron:

- No fue un movimiento unitario ni homogéneo.

- $\quad$ En él se encontraban varias vertientes de planteamientos societarios.

- $\quad$ Sobresale la postura modernizadora del Trabajo Social.

- Había interés por desarrollar una ruptura con los planteamientos conservadores que la caracterizaban.

- Tuvo influencias de procesos contestatarios de las clases subalternas que se dan en América Latina entre 1959 y principios de 1970, entre los que vale señalar la Revolución Cubana (1959), y la situación de Chile con la llegada a la presidencia del socialista Salvador Allende.

- $\quad$ Fue un proceso truncado por las condiciones impuestas en América Latina.

- $\quad$ En Costa Rica se presenta un planteamiento deformado del marxismo como referente teórico-práctico, especialmente por la lectura estructuralista francesa y la obra de Mao Tse-Tung.

- La Escuela de Trabajo Social en ese contexto, asume una política de "neutralización", para minimizar la fuerza de ese marxismo en Costa Rica, mismo que no era insumo teórico de la formación de la época.

- $\quad$ En Costa Rica, a pesar de que algunos movimientos populares se dieron a fines de la década del 60 y principios, del 70, tal movimiento quedó más bien en el ámbito universitario, el cual tendió a aparecer como dominante, en algunos sectores académicos.

Por su lado, Guzmán (1973) afirma que en la Reconceptualización se empezó a gestar una conciencia de cambio en el gremio y la academia latinoamericana, impulsándose principalmente por las agendas de los Seminarios Latinoamericanos de Servicio Social; igualmente destaca entre otros esfuerzos, los Documentos de Araxá y Teresópolis, ambos, como primer intento latinoamericano de sistematización del debate provocado por la Reconceptualización.

En esos documentos, se plantearon, según la autora, la necesidad de investigar, teorizar, enseñar y practicar un Trabajo Social latinoamericano, ello con el fin de estar en capacidad de dar una respuesta concreta a sus problemas regionales.

Cabe citar que las condiciones del continente eran difíciles y sangrientas, en Costa Rica particularmente existían ya algunas condiciones de persecución política más explícita, mismas que se habían gestado con José Figueres y el Partido Liberación Nacional desde mediados del siglo XX al amparo del artículo 98 de la Constitución de 1949; el cual, prohibía la organización comunista, por lo que la base de organización de izquierda tenía considerables limitaciones de desarrollo histórico.

Las mismas crisis generadas por las dictaduras de la época en Latinoamérica, posibilitó a pesar de todo, que en Costa Rica se pudieran recibir exiliados (as) y refugiados (as) de diferentes ramas académicas, los trabajadores y las trabajadoras sociales fueron parte de ellos/as.

$\mathrm{Al}$ respecto Retana destaca: 
La mayor influencia de este proceso en el caso de Costa Rica provino de Chile y de la Argentina, en razón de recibir exiliados por la dictadura militar en esos países: Entre ellos Diego Palma y Teresa Quirós que en 1977 se incorporan a la Universidad de Costa Rica (Retana, 2002, p. 69).

A pesar de esos aportes académicos, y aún sintiéndose las bases teórico metodológicas del funcionalismo norteamericano, con la participación ideológica y doctrinal de la Unión Católica Internacional de Escuelas de Servicio Social, la formación en estudio no logró, en el contexto de la Reconceptualización (inclusive por las propias contradicciones de la misma), romper ese lastre conservador que le dio vida.

Existieron intentos y debates, pero décadas después, se regresa a los causes del neopositivismo y las posturas modernizadoras del neofuncionalismo y algunas de sus expresiones, especialmente la teoría sistémica y de sistemas complejos.

Igualmente, la preocupación por la supuesta separación de la teoría práctica (herencia claramente funcionalista), parecía ser el eje de las preocupaciones de quienes se encontraban luchando por un ejercicio profesional más articulado a las demandas de intervención y de las expresiones de la "cuestión social".

A pesar de lo anterior, puede expresarse que paulatinamente, se van incorporando otras preocupaciones en la formación; sobre ese particular Picado (2003) detalla lo siguiente:

- En los años sesentas y setentas el objeto de la profesión cambia, se busca la liberación del hombre y la transformación de la sociedad.

- Trabajo Social dentro de su plan de estudios, empieza a visualizar el desarrollo de la comunidad, la interdisciplinariedad, las teorías funcionalistas del desarrollo, las teorías del cambio social.

- Los agentes profesionales se colocaban como agentes del cambio social.

- Aparece la Reconceptualización que viene con una influencia del llamado
Cono Sur, pero también prevalecen teorías de corte positivista sobre todo en la investigación.

- Dentro de las principales orientaciones en la formación en esos años se encuentran las de agente de cambio social, organizadores y promotores del desarrollo comunal.

- La orientación reconceptualizada del Trabajo Social buscaba movilizar, capacitar y generar participación.

- Había claridad en que se tenían que transformar las situaciones en que vivíamos, lo que no estaba claro era el manejo epistemológico y metodológico.

- Se incorpora la metodología de taller, aprender haciendo, aunque muchas veces en el camino las experiencias se estacionaban en el hacer y poco se aprendía de ese hacer.

Otra lucha que acompaña la formación de esos años, fue el que la unidad académica en estudio se incorporó a la recién formada Facultad de Ciencias Sociales ${ }^{4}$ (UCR); al respecto, el sociólogo Daniel Camacho - primer Decano de dicha facultad-, escribe sobre este tema lo siguiente:

La Facultad de Ciencias Sociales de la Universidad de Costa Rica fue fundada en el año de $1974^{5}$ [...] También se agregaron las Escuelas de Trabajo Social y Ciencias Políticas que funcionaban como anexas, la primera de la Facultad de Ciencias Económicas y la segunda de la Facultad de Derecho [...] Se encontraban ahí reunidas desde escuelas destinadas a un trabajo en el campo de la ciencia básica como puede ser la sociología, junto con escuelas dedicadas al trabajo práctico interdisciplinario como es el caso del trabajo social (Camacho, 1994, pp. 7 y 8).

Como se aprecia en la cita anterior, para el primer Decano de la Facultad de Ciencias Sociales, Trabajo Social es un "trabajo práctico interdisciplinario"; lo cual, retoma un poco la percepción que tenían algunas autoridades universitarias sobre la profesión, no siendo originariamente reconocida como ámbito de producción de conocimiento para las llamadas "Ciencias Sociales", sino más bien con un destino practicista de la "teoría de otros". 
Para $1973^{6}$, se plantea un nuevo currículo -y una nueva estructura académica ${ }^{7}$, léase al respecto:

Con relación a lo que se realizaba en años anteriores, se buscaba dar mayor participación a los sujetos a quienes se dirige la acción, a través del trabajo con grupos -generalmente son grupos existentes tales como Asociaciones de Desarrollo Comunal, Comités de Deportes, Jóvenes, etc., y/o a la formación de grupos encaminados a satisfacer las necesidades inmediatas de la población -donde los participantes aportan sus ideas y colaboran con las gestiones y acciones programadas. Es así, como si bien a nivel teórico, se llega a cuestionar la estructura social vigente, con la práctica se desarrollan estrategias orientadas a la acción y motivación de unidades reducidas pretendiendo con ello efectos multiplicadores que se extendían al resto de la sociedad. Lo anterior, a pesar de que no explicita totalmente lo que se plantea en la realidad, constituye un esfuerzo al interior de la Escuela que por un lado, posibilita los cambios posteriores en la estructura académica, y por otro acentúa la desvinculación total con las instituciones de Bienestar Social (Campos et al, 1977, p. 178).

Pero no sólo existieron preocupaciones nacionales para variar la forma- ción profesional, según destacan Molina y Romero (1992), luego del III Seminario Latinoamericano de Escuelas de Servicio Social (Quito, Ecuador, 1971), en la Universidad de Costa Rica, se iniciaron actividades académicas que se convierten en foros de discusión y análisis de las situaciones que se vivían en América Latina, señalan las autoras:

La Escuela toma contacto con el proceso de reconceptualización, y formalmente reconoce la necesidad de que, para poder formar un profesional de acuerdo con los tiempos, es preciso adecuar el currículo. Todos los aspectos curriculares son puestos en cuestionamiento y se ofrece una alternativa de cambio. Se propone partir de una concepción de hombre más realista, acercar el contenido formativo a las características del país y elevar el nivel científico. En síntesis se adhiere al proceso de reconceptualización del Trabajo Social (Molina y Romero, 1992, p. 66).

Algunos de los rasgos de la formación para 1973, se localizan en el siguiente cuadro:

Cuadro 8

Plan de Estudios, 1973

Escuela de Trabajo Social UCR

\section{Objetivo de la profesión:}

La liberación: proceso orientado a lograr que las personas o grupos conozcan su realidad y que se ubiquen en ella, la analicen y la critiquen, se equipen de instrumentos y técnicas que les permitan transformarla promoviendo los cambios estructurales para lograr su liberación.

\section{Propósito de la Escuela:}

Formar profesionales cuyas características esenciales han de ser su alto nivel científico ${ }^{8}$ y técnico, con vocación hacia la libertad cuyo papel había de ser el de participar con otros profesionales y con el pueblo, en el estudio y la superación de las contradicciones de la sociedad.

Buscar una mayor participación de la Escuela como un todo, los estudiantes y los profesores, en los procesos tendentes a formar una conciencia crítica en torno a los problemas del subdesarrollo y la dependencia, a remover los factores productores del hambre, la miseria, la ignorancia y la explotación de nuestro pueblo.

\footnotetext{
Organización Curricular:

a) Bloque de Cursos de:

1. Realidad nacional: que contribuyeron a la explicación de las causas estructurales de la pobreza y a un despertar de la conciencia crítica del estudiantado.

2. Metodología: la enseñanza hizo énfasis en el método básico ${ }^{9}$, que integraba los métodos de caso, grupo y comunidad con la idea de "entender al hombre en todas las dimensiones de su actuar"10"

1. Práctica: énfasis en trabajo de comunidades urbanas marginales.

b) Bloque subsidiario con cursos de: Psicología, Sociología, Administración y Legislación.
} 
Es vital resaltar que en $\mathbf{1 9 7 3}$ existió una preocupación por integrar el llamado "método dialéctico ${ }^{11}$ " en la formación profesional, ello como una supuesta respuesta a la necesidad de "aplicar la teoría en la práctica"12; al respecto se destaca textualmente lo siguiente:

Los cambios anotados en el objeto de la profesión, el propósito general de la Escuela y la estructura académica evidencian un problema real que anteriormente no se había planteado como tal, nos referimos a la separación entre los contenidos teóricos que se orientan hacia la transformación de la realidad y las posibilidades reales de aplicar el método dialéctico con fines de una verdadera praxis. Es así como la separación entre teoría y práctica se hace evidente (Campos et al., 1977, pp. 182 y 183).

El plan de estudios de $1973^{13}$ incluye por primera vez (producto también de las transformaciones que vive la Universidad de Costa Rica generadas por los Congresos Universitarios) los cursos de actividades culturales, deportivas, guía académica, matemática de ingreso, antropología (general y cultural), seminarios de realidad nacional, planificación económica y social de Costa Rica (dos cursos); dentro de los cursos tradicionales se encontraban castellano, filosofía (dos cursos, uno optativo en licenciatura), historia de las instituciones, repertorio de ciencias biológicas, sociología (rural, urbana y para el cambio social), economía (dos cursos), psicología (cuatro cursos), estadística (dos cursos), métodos de investigación, organización económica y social, metodologías del Trabajo Social (seis cursos), prácticas (seis cursos), administración general y de programas de bienestar social, ética, psiquiatría, derecho general y medicina social.

En la licenciatura luego de cinco años de cursos, se debía presentar y defender una tesis de grado de acuerdo a los reglamentos de la Universidad en mención.

Para 1974 se celebra en San José, Costa Rica, el IV Seminario de Escuelas de Servicio Social, condición que posibilitó que se pensara en elaborar otro nuevo plan de estudios, este evento giró entorno al tema "Organización y sistematización de las prácticas profesionales" 14 .

Pasados dos años (1976), se vuelve a establecer otro plan de estudios, el cual tiene la particularidad histórica que incluye El Taller como una estrategia pedagógica, tema sobre el cual no se puede profundizar en estas breves reflexiones, pero se espera retomar en próximos artículos.

Prosiguiendo con el análisis, vale señalar que es hasta ese año ${ }^{15}$ (1976), según Campos et al. (1977), que la Escuela establece una propuesta de plan de estudios donde la academia de Trabajo Social (producto de las tres primeras Jornadas de Trabajo Social) ubica concretamente la situación del país y de la profesión en él, léase al respecto:

1.1 Costa Rica: país subdesarrollado. Situación de dependencia externa (grupos populares). 1.2 En nuestro país esta dominación se ejerce principalmente a través de la educación, las políticas de bienestar social, los medios de comunicación. 1.3 Tal situación se manifiesta mediante el análisis de las estructuras agrarias e industriales, la estructura de poder y la de las clases sociales. 1.4 La situación anterior transforma en un objeto dominado ( las características negativas de éste no se aprecian como resultado de causas estructurales; hay separación de efecto y causas) 1.5 Frente a esa realidad se asume la necesidad de su transformación (liberación del hombre) [...] 2.1 Partiendo de esos elementos se puede definir el Trabajo Social (trabajadores sociales) como un agente que, siendo externo, se inserta en la situación concreta para colaborar en la creación de condiciones más racionales que favorezcan la toma de conciencia, por parte del hombre dominado, su capacitación y organización (su movilización, en resumen) 2.2 La acción concreta convenientemente fundamentada, reflexionada y sistematizada, permite lograr un conocimiento más dinámico y profundo sobre la realidad social" (Campos et al, 1977, Anexos VII-5).

El sustento teórico metodológico para la intervención profesional, al menos planteado en ese entonces, sugería partir de lo siguiente:

a) de un concepto de hombre según el cual éste es sujeto de su transformación y del mundo en que vive. b) de una concepción de realidad como unidad dinámica (dialéctica en el sentido de que constante cambio se genera, a partir de la oposición de los elementos que se dan en situaciones concretas y de acuerdo a 
ciertas leyes) c) de una concepción del conocimiento como proceso que se da a partir del elemento sensorial y concreto para llevar a la elaboración abstracta y luego, a una interpretación teórica de la realidad concreta (Campos et al., 1977, Anexo VII-5).
Para los años siguientes, los fines de la Escuela plantean cambios en su rumbo, léase al respecto:

Cuadro 9

Plan de Estudios

$\left(1976^{16}-1981^{*}\right)$

Objeto de Estudio: Se estudian los grupos sociales que presentan un marcado deterioro en Costa Rica, principalmente los populares, que por ser históricamente los grupos de atención mayoritaria, cualitativa y cuantitativamente de la profesión, obligan a estudiantes y profesionales en Trabajo Social a manifestar compromiso o identificación con ellos.

Objetivos de la Escuela: Formar un profesional con capacidad para comprender científicamente la realidad y para colaborar en los procesos de transformación social estableciendo relaciones dialógicas con los grupos populares, y que ejecute acciones de asistencia y promoción social conjuntamente con otros profesionales, atienda situaciones de asistencia y promoción social conjuntamente con otros profesionales; asesore, planee, ejecute y evalúe acciones, proyectos y programas de Bienestar y Desarrollo Social; diseñe y realice investigaciones sociales y supervise personal asignado a programas de Bienestar Social.

Organización Curricular: Método de Taller, el cual es un proceso de enseñanza-aprendizaje de carácter social: en el que se transmite la información y síntesis del desarrollo científico de la humanidad. Se organiza curricularmente en forma de unidades integradas de conocimiento, extraídas de la realidad social y abordadas desde la perspectiva científica, que partiendo de la experiencia empírica (práctica académica) reconoce un camino que va de lo simple a lo múltiple para retomar la realidad y contribuir a su transformación.

La organización curricular presupone la integración de tres niveles: integración teoría-práctica, integración docencia-investigación y acción social, integración docente-estudiante-grupos sociales.

Contenidos teóricos y prácticos en tres áreas temáticas: área de Teoría Social, área de Realidad Nacional, área de Teoría y Metodología del Trabajo Social.

* $\quad$ El Plan de Estudios sufre modificaciones de tipo administrativo que se aprueban en el año de 1981. Fuente: Molina y Romero, 1992.

A lo largo de este último apartado, se logró identificar, cómo la influencia histórica del continente latinoamericano, generó presiones sobre la formación profesional; el llamado Movimiento de Reconceptualización, fue prueba de esa complejidad.

Las expresiones y resistencias hacia los rumbos que se tomaban para responder a esas condiciones fueron particulares y singulares en cada país, en el caso costarricense, pareció ser una base para replanteamientos curriculares, didácticos y pedagógicos.

La formación, también entró en este período en un espacio de madurez académica, donde su interlocución con otras áreas por medio de la creación de la Facultad de
Ciencias Sociales, es un hecho de suma relevancia.

Otro de los insumos que caracterizan esos años y que aún debe investigarse, es la llamada influencia de la dialéctica, como sustento teórico-metodológico.

Sin duda, el aparecimiento del Taller como estrategia pedagógica que marcó el rumbo de lo que antes eran los espacios de práctica, es un eje base del estudio crítico de la época, hasta el presente.

Estos años, también dan evidencia de la necesidad de espacios sostenidos de autocrítica y crítica de la formación, insumo que permitió, en alguna medida, ventilar cuestiones que se requieren mejorar, desde luego, bajo una cierta orientación hegemónico académica. 


\section{d) A modo de cierre:}

La madurez de la formación profesional en Trabajo Social, se ha ido forjando entre procesos con intentos de ruptura, impulsada también por una fuerte orientación de dar respuesta a las demandas del contexto nacional y latinoamericano.

La incorporación de la Escuela de Trabajo Social a la UCR, fue, con las críticas que correspondan, un impulso de crecimiento académico, con resultados relevantes, en comparación a otros países donde existió durante décadas, sin rango de educación terciaria y con perfil tecnicista.

La formación en Trabajo Social ha demostrado ser objeto de análisis y discusión desde la década de 1940, llevando a constantes cambios y replanteamientos, aunque no necesariamente a rupturas, con sus bases fundacionales norteamericanos (especialmente de matiz clínico o terapéutico); prueba de ello se localiza en sus planes de estudio iniciales.

Las nociones de "suma de partes", "profesión complemento", o "práctica de la teoría de otros", incorporadas en algunos tramos de los planes de estudio, fue generada por las limitaciones académicas e investigativas que se caracterizaban en la época; mismas que se reducían a tener como foco de atención, preocupaciones teórico-practicistas, centradas en la tradicional trilogía metodológica de caso, grupo y comunidad, y a su vez, la búsqueda de la aplicación de la "teoría a la práctica".

El papel de la ONU en los inicios del desarrollo de la formación, fue relevante, y poco estudiada, sin embargo, se logran identificar críticas hacia sus lineamientos, generándose sentimientos de autonomía, que posibilitaron un desarrollo académico más vinculado con el entorno universitario, y la cuestión de la institucionalización de las garantías sociales (trasfondo constitucional de la profesión), así como otras formas de intervención en la "cuestión social" en Costa Rica.

Sin embargo, Costa Rica y muchos otros países de Latinoamérica, no dieron muestras de romper con las bases del Trabajo Social tradicional norteamericano, aunque académica y gremialmente hubo esfuerzos; al parecer, la hegemonía estaba en una colectividad formada bajo fundamentos tradicionales y conservadores, que identificaban esos planteamientos como una amenaza personal (vulnerabilizaba sus bases) o de clase (a pesar del discurso de anticapitalismo romántico de la época).

En todo el trayecto histórico analizado, el desvelo por la preocupación por la integración inmediata de la teoría y la práctica, así como el llamado a sistematizar para "producir teoría", fueron características en el desarrollo de la formación académica de la época, lo cual, llevó especialmente en los años setentas, a caer en la trampa de considerar que un llamado "método dialéctico", iba a ser el catalizador entre esa demanda y la crítica al Trabajo Social tradicional.

Sin embargo, la historia demuestra que esa salida lateral, no tocaba los elementos de fondo, ya que aunque la crítica pudiera ser considerada una cuestión del claustro académico, y que al interior de la universidad se expresaran obstáculos a ese impulso de cambio, el gremio en su totalidad no tenía las condiciones históricas (objetivas y subjetivas) para una ruptura con los elementos sincréticos que fundaron a la profesión en el país.

La tendencia modernizadora, la separación predominante de prácticas pre profesionales fuera del mundo de trabajo gremial (bajo los llamados “Talleres"), las iniciales preocupaciones epistemológicas, y la crítica a la trilogía metodológica (caso, grupos, comunidad), derivando en otras propuestas como el método integrado, el método único y el método polivalente; así como un pseudomarxismo, fueron las cuestiones más complejas que se localizan en el convulso período formativo de los años en estudio.

En resumen, la formación académica de este período se caracterizó por una constante de cambio, una búsqueda de respuestas a demandas histórico-contextuales 
que implicaban a la profesión, así como al planteamiento de diferentes caminos (algunos antagónicos).

Esas décadas evidencian la complejidad del tema educativo en Trabajo Social, en tanto su constitución teórica y filosófica, como las demandas de método que subyacen por la dialéctica histórica de la que forma parte.

Los siguientes períodos, entre ellos la llamada "década perdida" de los años ochentas, son deudores de las fuerzas históricas derivadas de la búsqueda de un referente más latinoamericano, más costarricense, más crítico, pero a su vez, más compleja, tanto en su dimensión ideológicopolítica como teórico-metodológica.

Volviendo la mirada a los inicios del siglo XXI, es posible evidenciar que la trayectoria académica de la Escuela de Trabajo Social, sigue mostrando sus avances en materia formativa.

Por ejemplo, fue una de las primeras unidades académicas que recibió la acreditación de su plan de estudios, tanto a lo interno de la UCR, como del país.

A partir del año 2004, realizó una interesante reforma curricular basada en los debates críticos más contemporáneos en el ámbito latinoamericano, y con influencia de algunas tradiciones críticas derivadas desde el pensamiento marxista clásico, la Escuela de Frankfurt, la Escuela de Budapest, la llamada "nueva izquierda" británica, y los aportes de investigación generados por programas de posgrado (maestrías y doctorados en Trabajo Social, de Brasil, Argentina, Uruguay y Chile).

Vinculado a ello, se encuentra su actual propuesta de gestar núcleos de estudio, investigación, extensión y docencia, dirigidos a ciertas expresiones de la "cuestión social" en Costa Rica, así como para fundamentar algunos ejes básicos de la estructura curricular.

Entre los principales desafíos en materia de formación académica, es posible señalar que la Escuela, se ha comprometido con una serie de medidas que tocan puntos críticos tales como: lograr fortalecer la preparación para la investigación, consolidar en la formación una serie de insumos ético políticos, mejorar los espacios de práctica pre profesional, establecer una estructura curricular que le permita al estudiante poder trabajar en tiempo de clases, aumentar los niveles de estudios de los docentes a grado doctoral, acortar los tiempos de graduación, investigar el mercado de contratación y las demandas de competencias $\mathrm{y}$ atribuciones que den mayor reconocimiento a sus egresados en nuevos espacios de contratación, consolidando a su vez los tradicionales.

\section{Notas}

1. Para Guzmán (1973) estos textos eran traducciones al castellano de las obras norteamericanas, lo que llevó a una referencia teórica de Estados Unidos, en un país tan distinto.

2. Un evento que posteriormente tiene impacto en la formación, es que para el año 1960 la Escuela es designada como Sede del IV Congreso Panamericano de Servicio Social, y en el año de 1962 se integra a la Asociación Internacional de Escuelas de Servicio Social (Mora, 1968).

3. La fuente original no fue posible de localizar en la actualidad.

4. Lo que significó una disminución en la injerencia hacia la Escuela por parte de las instituciones estatales en la determinación de sus políticas, Campos et al., 1977. Vale anotar que antes de crearse la Facultad de Ciencias Sociales, se había pensado en una Escuela de Ciencias Sociales, pero el proyecto no fructificó (Mora, 1968).

5. Continúa el autor: La constitución de esta Facultad fue uno de los productos del tercer congreso universitario [...] Existía antes del tercer congreso universitario La Facultad Central de Ciencias y Letras, la que fue el principal producto de la Reforma universitaria de 1957 liderada por Rodrigo Facio, Carlos Monge Alfaro, José Joaquín Trejos, Enrique Macaya Lahman y otros prohombres universitarios [...] Sin embargo se impuso la idea de dividir la Facultad de Ciencias y Letras 
de la forma dicha. Es así como se constituyó la Facultad de Ciencias Sociales. A ella se incorporaron las Escuelas de Historia y Geografía y Ciencias del Hombre (Camacho, 1994, pp. 7 y 8).

6. "La preocupación fundamental de estos años fue el discutir lo que se hace y definir lo que debe hacer el profesional en Trabajo Social, así como relacionar ambos aspectos con la historicidad [...]" (Campos et al., 1977, p., 173).

7. En ese momento se encontraba conformada por un Consejo de Profesores como máxima autoridad, una persona como Directora con funciones ejecutivas, un Consejo Directivo (llamado en 1964 Consejo Asesor) compuesto por quien tiene la Dirección, las personas responsables de las secciones de investigación (quien inició como departamento y tenía a su cargo reorganizar el sistema de tesis y examen de grado, la creación de un reglamento de investigación, así como tener una postura crítica ante la "metodología" y técnicas de investigación que se realizan), acción social (a petición de los estudiantes y las estudiantes se crea en 1972 como Comisión de "Relaciones Públicas" con el fin de dar a conocer la profesión, pero básicamente era la encargada de la proyección de la Escuela), práctica (se origina por la necesidad de supervisar la práctica que se propone en el Plan de 1965) y docencia (la cual pretendía lograr que a través de los bloques de cursos se respondiera a un análisis más acertado de la realidad, velaba por el mejoramiento continúo de planes y programas), así como la representación de dos estudiantes. Existía además un equipo interdisciplinario formado por profesores de varias profesiones como Trabajo Social, Sociología, Filosofía, Psicología, Historia y Economía que evaluaban la práctica (Campos et al., 1977).

8. Se destaca al respecto: "Los fundamentos epistemológicos explicitaron las bases del materialismo histórico y dialéctico en consonancia con los postulados del movimiento de reconceptualización. (Molina y Romero, 1992, p. 67) (Cursiva del original)

9. La inclusión del llamado método básico, de origen chileno (Retana, 2001), ha sido una de las mayores críticas que se le pueden hacer a esta experiencia reconceptualizadora en Costa Rica, ya que la misma no tiene distanciamiento teórico-metodológico con los ya tradicionales caso, grupo y comunidad en términos de esencia.
10. También se partió de la orientación educativa llamada educación popular, teniendo entre sus expositores al brasileño Paulo Freire.

11. Señala Quiroga (1990) lo siguiente: "Es interesante notar que ese proceso no rompió con una herencia conservadora, de cuño positivista e irracionalista, predominante históricamente en el Servicio Social, lo que va a constituirse en uno de los factores explicativos de la aproximación tardía de la profesión al debate del marxismo y a su incorporación por vía de un marxismo positivista, suavizado en los moldes de Althusser y Harnecker", pp. 132 y 133).

12. Pudiendo caer por supuesto en un funcionalismo radical.

13. Este mismo año se celebró en Costa Rica el seminario "Trabajo Social y Población" con participación de México, Centroamérica y Panamá, donde su comité organizador estuvo compuesto por Lic. Carlos María Campos por parte de la Caja Costarricense de Seguro Social, Lic. Olda Acuña de la Asociación Demográfica, Lic. Luis Fernándo Mayorga de la Escuela de Trabajo Social de la UCR, Lic. Yolanda Mendoza del Centro de Orientación Familiar, Lic. Flor López del Ministerio de Salud Pública y la Lic. Elsa González como representante del Colegio de Trabajadores Sociales. Fuente: Boletín de información para los/as trabajadores/as sociales Año 21973. Colegio de Trabajadores Sociales de Costa Rica. San José, Costa Rica.

14. Se contó con participación de profesionales de Argentina, Brasil, Chile, Colombia, Ecuador, Panamá, Guatemala, Honduras, El Salvador, México, República Dominicana, Perú, Venezuela y Costa Rica. Entre los/as costarricenses que presentaron ponencias se encontraron Guzmán, Laura; Mejía, Luz y Jiménez, Kembly con el trabajo: "Proposiciones para la reformulación del "Plan de Acción" de la Escuela de Trabajo Social”. También expusieron Odio, Marta y Delgado, Florisabel "Primera aproximación a la sistematización de las experiencias prácticas de la Escuela de Trabajo Social”; Quirós, Tito; Montejo, Athenia; Quirós, Teresa y Acuña, Víctor con la ponencia. "Hacia un nuevo tipo de Escuela de Trabajo Social"; Rosendorn, Haydee con: "Utilización de métodos pedagógicos que implican algún tipo de innovación” y Palma, Diego (profesor extranjero radicado en Costa Rica) con "Sistematización". Igualmente en este evento la presidencia de ALAETS-CELATS, 
queda bajo la presidencia de Beatriz de la Vega (México), y la costarricense Florisabel Ramírez fue reelecta como vicepresidenta, y la representación estudiantil a cargo de María Atilano (El Salvador). Fuente: Boletín de Información para Trabajadores/as Sociales. Año 3, Número 14. Diciembre de 1974.Colegio de Trabajadores Sociales de Costa Rica. Aunado a ello, en esa actividad se aprueba, por propuesta de la Escuela de Trabajo Social de la Universidad de Costa Rica, la suspensión de las escuelas de Trabajo Social chilenas, que fueron criticadas por su posición ante las políticas de la dictadura de Augusto Pinochet; lo anterior se resume en el siguiente pronunciamiento: 1- Las Escuelas de Trabajo Social de Chile, quedan a partir de esta fecha, excluidas como miembros de la Asociación Latinoamericana de Escuelas de Servicio Social (ALAESS), hasta que en su país no se restituyan las garantías para el ejercicio profesional, cesen las violaciones a los derechos humanos y que a la Universidad permita una expresión científica. 2- Elevar a las embajadas de Chile de los respectivos países una solicitud de libertad de los Trabajadores Sociales chilenos presos y convertir el movimiento liberador en un movimiento latinoamericano. 3- Repudiar la actitud del Colegio de Asistentes Sociales de Chile, quienes delataron y vendieron a sus colegas por no pensar como La Junta de Gobierno, siendo muchos de estos Asistentes Sociales, exonerados de sus trabajos, encarcelados, torturados o fusilados (CTS, 1974, 40).

15. En este año se celebra el Seminario sobre Trabajo Social en el Campo del Bienestar Estudiantil en las Instituciones de Enseñanza Superior. Boletín Informativo, Colegio de Trabajadores Sociales de Costa Rica N. 19, 1974.

16. Entre los planteamientos generales de la Escuela para 1976, se destaca con más detalle lo siguiente: Fin: Formación de profesionales definida por la realidad en que está inserto/a y por los fines propios de la Universidad. Propósitos generales: 1-Desarrollo de la capacidad para conocer y comprender la realidad de forma científica (en su dinamismo y unidad). 2-Desarrollo de habilidades para crear y utilizar métodos, técnicas e instrumentos que permitan colaborar en los procesos de transformación social. 3-Desarrollo de actitudes que lleven a comprometerse responsablemente en la realización de acciones concretas mediante el establecimiento de relaciones dialógicas con los sectores populares. 4Desarrolle la colaboración en tareas concretas a partir de grupos, comunidades, organizaciones y/o instituciones, siempre que éstas tengan significación en términos de los fines de la transformación social. Conocimientos y Comprensiones: 1-Realidad costarricense, dentro del contexto latinoamericano y mundial. 2-Del Trabajo Social en su relación actual y en su proceso histórico (ubicación en la realidad y formas de enfrentarlas). 3-Elementos de la Ciencia Social que ayudan a una interpretación de esa realidad. Habilidades: 1-La observación, comprensión e interpretación científica de los fenómenos sociales a partir de situaciones concretas (relación de lo concreto con lo abstracto; del particular con lo general). 2-Para definir políticas generales, formular y ejecutar planes, de acción (definición de fines, objetivos, estrategias y tácticas, toma de decisiones y trabajos en equipo). 3-Para la aplicación creativa y racional de métodos, técnicas e instrumentos propios del Trabajo Social (concienciación, capacitación, organización y gestión). 4-Para la sistematización de la experiencia práctica. Actitudes: Realizar su acción conjuntamente con los grupos populares mediante una relación racional, responsable y dialógica. Fuente: Campos et al., 1977, Anexo VII-5.

\section{Referencias bibliográficas}

Araya, R., Calderón, M., Chinchilla, J. y Vega, C. (1986). Análisis del surgimiento y desarrollo del Servicio Social en Costa Rica y su ubicación en el contexto económico social y político. Informe Final de Proyecto de investigación. Carrera de Trabajo Social. Sede Universitaria de Occidente, Universidad de Costa Rica. Costa Rica.

Camacho, D. (1994, Junio). Génesis y primeros pasos de la Facultad de Ciencias Sociales. Revista de Ciencias Sociales. Universidad de Costa Rica, 64, 7-10.

Campos, I. (1992). La práctica académica de la Escuela de Trabajo Social: 19421990. Revista de Ciencias Sociales. Universidad de Costa Rica, 56, 51-61.

Campos, I., Molina, E., Molina, M. L., Romero, C. y Ruiz, A. (1977). Evolución social de la estructura académica de la Escuela de Trabajo Social en Costa 
Rica. Tesis de licenciatura para la obtención del título de Licenciadas en Trabajo Social. Escuela de Trabajo Social. Universidad de Costa Rica. Costa Rica.

Colegio de Trabajadores Sociales de Costa Rica. (1974). Boletín de Información para Trabajadores/as Sociales,14, Año 3, 12.

Comisión de Autoevaluación y Autorregulación de la Carrera de Trabajo Social. Universidad de Costa Rica. (1998). Proyecto para la autoevaluación y autorregulación para la acreditación de la carrera de bachillerato y licenciatura en Trabajo Social. San José, Costa Rica: Escuela de Trabajo Social.

Esquivel, F. (2007). Estudio exploratorio sobre los fundamentos orgánicos de la formación académica en la Universidad de Costa Rica. Tesis de doctorado para la obtención del título de Doctor en Educación. Doctorado Latinoamericano en Educación. San José, Costa Rica: Universidad Estatal a Distancia.

Esquivel, F. (en prensa). Desarrollo capitalista en el estado empresario en Costa Rica: aportes para el Trabajo Social. Revista Reflexiones.

Gramsci, A. (1999). Antología. México: Editorial Siglo XXI.

Gurdián, A., Guzmán, L., Molina, M. L. y Romero, M. (1992). Planificación y evaluación curricular en Trabajo Social. San José, Costa Rica: Editorial Alma Máter.

Guzmán, L. (1973). Una estrategia para el cambio en el Trabajo Social costarricense y de la sociedad: aspectos tácticos, metodológicos y estructu- rales. Tesis de licenciatura para la obtención del título de Licenciada en Trabajo Social. Universidad de Costa Rica, San José, Costa Rica.

Molina, M. L. y Romero, M. (1992). La planificación y la evaluación curricular, la experiencia de la Escuela de Trabajo Social de la Universidad de Costa Rica 1942-1991. San José, Costa Rica: Editorial Alma Máter.

Molina, M. L. y Sáenz, J. A. (1994). Trabajo Social: lo académico y lo profesional. Revista de Ciencias Sociales, 64, 101-108.

Mora, R. (1968). Algunos aspectos relacionados con la formación de profesionales en la Escuela de Servicio Social de la Universidad de Costa Rica. Tesis de licenciatura para la obtención del título de Licenciada en Ciencias Económicas con especialización en Servicio Social. Escuela de Trabajo Social. Facultad de Ciencias Económicas. San José, Costa Rica.

Netto, J. (1990). Ditadura e Serviço Social. São Paulo, Brasil: Cortez Editora.

Picado, M. (2003, 23 de abril). Desafíos Históricos del Trabajo Social en la Formación Profesional. Escuela de Trabajo Social. Facultad de Ciencias Sociales Notas de la exposición presentadas en el Foro: Desafios Históricos del Trabajo Social en los ámbitos de la salud, la educación, la justicia y la formación profesional. Universidad de Costa Rica. San José, Costa Rica.

Retana, J. (2002). As dimensões a ética e da política no Serviço Social: a particularidade a Costa Rica. Disertación para optar por el grado de Máster en Servicio Social. Río Janerio, Brasil: Universidad Federada de Río de Janeiro. 